\title{
A Multi Echelon Location-Inventory Model with Lateral Transshipment
}

\author{
Mohammad Reza Gholamian \\ School of Industrial Engineering, \\ Iran University of Science and Technology, Narmak, 16846-13114, Tehran, Iran \\ Email: Gholamian@iust.ac.ir (Corresponding Author) \\ Mustafa Nasri \\ School of Industrial Engineering, \\ Iran University of Science and Technology, Narmak, 16846-13114, Tehran, Iran \\ Email: Mostafa_nasri@ind.iust.ac.ir
}

\begin{abstract}
In this research, a supply chain system consisting producer, distribution centers and retailers is modeled by considering lateral transshipment between distribution centers and also using echelon-based inventory system instead of independent inventory system. The model is developed in the form of mixed integer non-linear programming (MINLP) to minimize the total location, transportation, and inventory costs of the system. The model was solved using conic programming approach and validity was examined by comparing the developed model with the basic model (i.e. the model without the contributions) in several instances with different sizes of distributors and retailers. The results represent superiority of the developed model in computational time and objective value especially in medium and large-scale problems.
\end{abstract}

Keywords: location-inventory, lateral transshipment, multi echelon inventory system, conic programming

\section{INTRODUCTION}

Generally, in most researches the inventory costs are modeled as a part of total cost of a single company at each layer of supply chain. But this modeling issue leads to neglecting different relations which may increase costs, reduce chain efficiency, and increase supply time (Shen et. al, 2003). Rather, in recent years, multi-echelon inventory systems are introduced in supply chains. In these systems, the concept of echelon stock is used instead of the concept of installation stock (Axsäter, 2015).

In traditional viewpoint, the inventory position of an installation (i.e. a firm or company in supply chain) at each layer is defined only based on the stock at that installation. But due to lack of systematic relationship between the installations at different layers, the ordering policy did not work properly. For example, while the stocks of installations in downstream layers are very high and we don't need to immediately replenish an installation in upstream layer to support downstream, the replenishment of this upstream installation is made only based on its low inventory position.

Now, suppose that the inventory position of an upstream installation is taking into account by also including downstream stocks. Then, the inventory position represents the real stock of an upstream item in all of the chain which is called echelon stock (Sherbrooke, 2004). Alternatively, the costs should be represented in terms of echelon stock. Since the echelon stock of an upstream item includes the stock of a downstream item, the holding cost of downstream item should only include the value added of producing from its upstream item; which is known as echelon holding cost (Axsäter, 2015).

Specifically, this consideration is more compatible with real-world operations of supply chains and at the same time optimize the model with much lower cost than traditional approaches (Hillier \& Lieberman, 2015).

Another disadvantage of traditional approaches is hierarchical definition of transportation flows from upper layer to lower layer; whilst using information technology, supply chain systems and subsequently inventory systems can be designed more flexible by allowing lateral transshipment within the layers. In this way, the peer installations of the same layer can share their stocks among each other to reach the lower inventory levels and also lower costs by still achieving the expected service level. There are many real cases of such transshipments in practice especially at peak working times such as Christmas which the transshipments can help in cost and inventory level reduction of distribution centers.

In this article, both above concepts are taken into account in a multi-layer supply chain model. It is assumed that inventory is only stored in active distribution centers. So, both multi-echelon inventory/safety stock costs and hierarchical/lateral transshipment costs are considered among distribution centers to make more precision and reality.

Based on above assumptions, the model was developed as a mixed integer nonlinear programming (MINLP). Since solving such models is generally very difficult, the model is transformed into its equivalent conic programming model and then the model is solved and the results were analyzed.

In summary, our contribution can be explained as follows:

1. Developing a supply chain network design by considering multi-echelon (instead of multi-level) location-inventory model. 
2. Adding lateral transshipment between distribution centers in a two-echelon supply chain network.

3. Solving the developed model using conic programming approach.

All three aspects will be reviewed in the next section. First, researches on conic programming approach have been reviewed. Subsequently, review of studies related to lateral transshipment are brought in detail and finally, few studies on echelon-based models have been presented.

The rest of the paper is organized as follows. Section 2 presents the review of literature on related studies and background of the developed models. In section 3 the proposed model is introduced. Section 4 deals with solution approach. Numerical results are brought in section 5 and finally section 6 concludes with summarization and future directions of the research.

\section{LITERATURE REVIEW}

As the first studies, Shen (2000) presented a locationinventory model in mixed integer nonlinear programming considering ordering, safety stock and holding costs in objective function and is recognized as one of the most popular researches of this area. Daskin et al. (2002) extended the model by adding transportation costs to the model and solving it using Lagrangian relaxation approach. Capacity limitation of distribution centers was considered by Ozsen $e t$ al. (2008) with the aim of risk reduction. In similar way, recently Maass et al. (2016) introduced a model which uses inventory buffers instead of capacity. Mak and Shen (2009) modeled the location-inventory problem considering radius coverage for retailers and allowing for inventory shortages in a queuing system. Berman et al. (2012) considered periodical review inventory policy for distribution centers and used Lagrangian relaxation method to solve the proposed model. Recently, Lagrangian relaxation was used in three-level distribution system by Ross et al. (2017) in a locationinventory model with safety stock and considering distribution coverage radius. To solve the model at first, the square root terms of objective function are replaced by adding new variables and constraints into the model and then relaxation was made on assignment constraints.

On the other hand, in conic programming applications, Atamtürk et al. (2012) used conic optimization approach for several supply chain models and demonstrated the performance superiority of this approach in comparison with other previous methods in terms of solution times and objective values. Shahabi et al. (2013) developed four-level supply chain model by considering hubs in the third level. The model optimized location, allocation and inventory decisions simultaneously. The safety stock is considered in the model based on service level type 1 and then the model has been solved using conic programming approach. Also, they extended their research by considering demand and lead time dependencies in the model and again conic programming with compact formulation was used as solution approach (Shahabi et al, 2014). Zhang and Unnikrishnan (2016) developed a closed loop supply chain with distribution centers in forward and reverse network under periodic review policy. The model is reformulated by first linearization of the product variables and then converting the chance constraint using conic programming. Finally, Puga and Tancrez (2017) proposed a heuristic algorithm for solving large-scale supply chain networks and compare their results with conic programming approach. The results demonstrate a few differences in optimality gap.

On the other hand, some studies developed supply chain network design involving lateral transshipment. Nasiri et al. (2015) proposed a two-level (plant-warehouse) model in which the warehouses are divided into "main" and "regular" categories and lateral transshipment from the main into regular warehouses is allowed. The model was solved using mimetic algorithm in medium and large-scale sample sizes. Ahmadi et al. (2016) developed a three-level supply chain network such that internal transshipment was considered for distribution centers in the second level. The model is developed in a mixed integer linear programming with fuzzy parameters and hence fuzzy solution approaches was used for solving the model. Daghigh at al. (2016) presented a sustainable multi-modal, multi-period, and multi-objective model in a 4-level network in which lateral transshipment is permitted in second and third levels. The model was developed with three objective function regarding to economical (i.e. minimum cost), social (i.e. maximum social responsibility) and environmental (i.e. minimum fuel consumption) aspects of sustainability. The model was solved using $\varepsilon$-constraint method.

Meanwhile specially, some recent studies have been developed based on dynamic programming approach. Paterson et al. (2012) proposed a location-inventory model in which the lateral transshipment is permitted when a location is faced with shortage. The model considered the stochastic demand with Poisson distribution and used dynamic programming as modeling and solution approach for only two locations. In another research, VanWijk et al. (2012) used lateral transshipment in a spare part inventory system. The model considered that stochastic demand with Poisson process which is responded either directly, or through another warehouse or even through emergence sources, each with different probability and then a Markov chain process model has been developed and solved based on these probabilities. They extended their research by adding the concept of quick response warehouse to the model as an intermediate level of supply chain (VanWijk et al, 2013). Recently, another extension of the model with two stock points was developed such that each stock point replenishes multiple groups of retailers ordered by their importance (VanWijk et al. 2018). In Haji et al. (2014) a supply chain with one supplier and two retailers was considered such that one of retailers has less Poisson demand than another one and can cover it in emergency conditions under (1, T) and (s-1, s) polices. Also, Olsson (2015) developed a METRIC ${ }^{1}$ (Sherbrooke, 2004) inspired method to modeling the inventory of two locations with lateral transshipments. Demand is defined based on Poisson process such that the intensity function is also a Poisson process (i.e. doubly Poisson Process). The method was evaluated by simulation and the results showed that the method performs very well. Feng et al. (2017) developed a multi-location inventory model with lateral transshipment such that an

\footnotetext{
${ }^{1}$ METRIC: Multi Echelon Technique for Recoverable Item Control
} 
approximate dynamic programming algorithm was used for solving the model. In fact, an approximate of value function is considered in decision states of dynamic programming approach. In similar way, Meissner and Senicheva (2018) introduced a multi-period, multi-location inventory model with lateral transshipment and stochastic demand. The model was developed using dynamic programming approach on a piecewise-linear approximation of objective function.

Unfortunately, there are rarely studies based on the multi-echelon inventory models. As near topics, Diabat et al. (2013) developed a two-echelon location-inventory model which is solved using Roundy's \%98 approximation method based on relaxation of assignment constraints into objective function. This research is completed by consecutively developing upper and lower bounds of Lagrangian relaxation to reach the optimal solution (Diabat et al, 2015) and also the model is extended into green supply chain by considering weighting factor of carbon emission into the model (Abu Alhaj et al, 2016). Meanwhile, piecewise linear approximation was used for linearization of non-linear parts of the model (Diabat and Theodorou, 2015). However, in the last research, the solution approach has been changed using capacity constraints instead of Roundy's "power of two" inventory policy and then the model is linearized using piecewise linear approximation (Diabat, 2016). Sadjadi et al. (2016) used queue theory to determine positive and negative inventory levels in a three-level supply chain model. Similarly, queue theory was used by Diabat et al. (2017) in determining expected reorder point, inventory and lost sale with uncertain demand and uncertain lead time.

In a broader view, Table 1 illustrates the summary of the most important researches in recent years. As shown in the Table, the multi-echelon inventory systems along with lateral transshipment have not been used in previous studies. On the other hand, the investigation of solution approaches demonstrates that there are a few applications of conic programming.

So as mentioned previously, in this study, we try to develop a real-world model by adding both multi echelon inventory system and lateral transshipment to the locationinventory model and then solving the developed model using conic programming approach. Finally, the developed model is evaluated by comparing the results with the model without these contributions.

Table 1 Summary of literature review

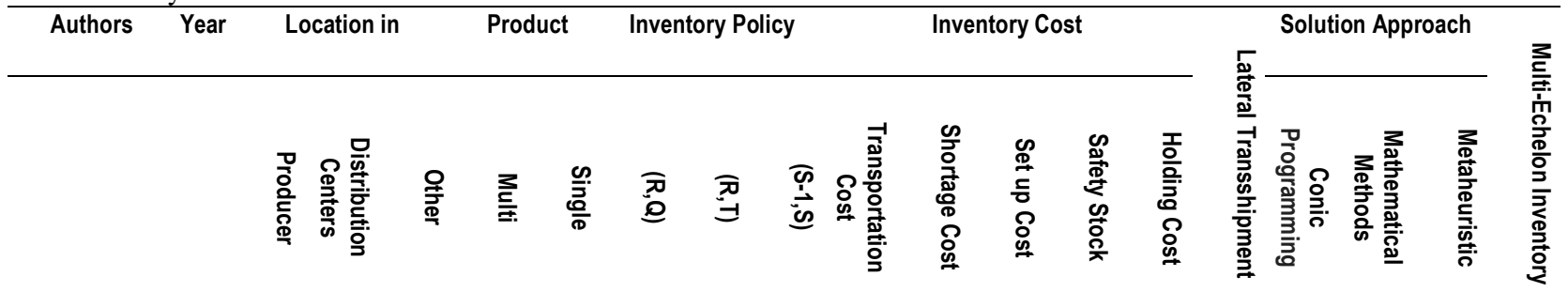

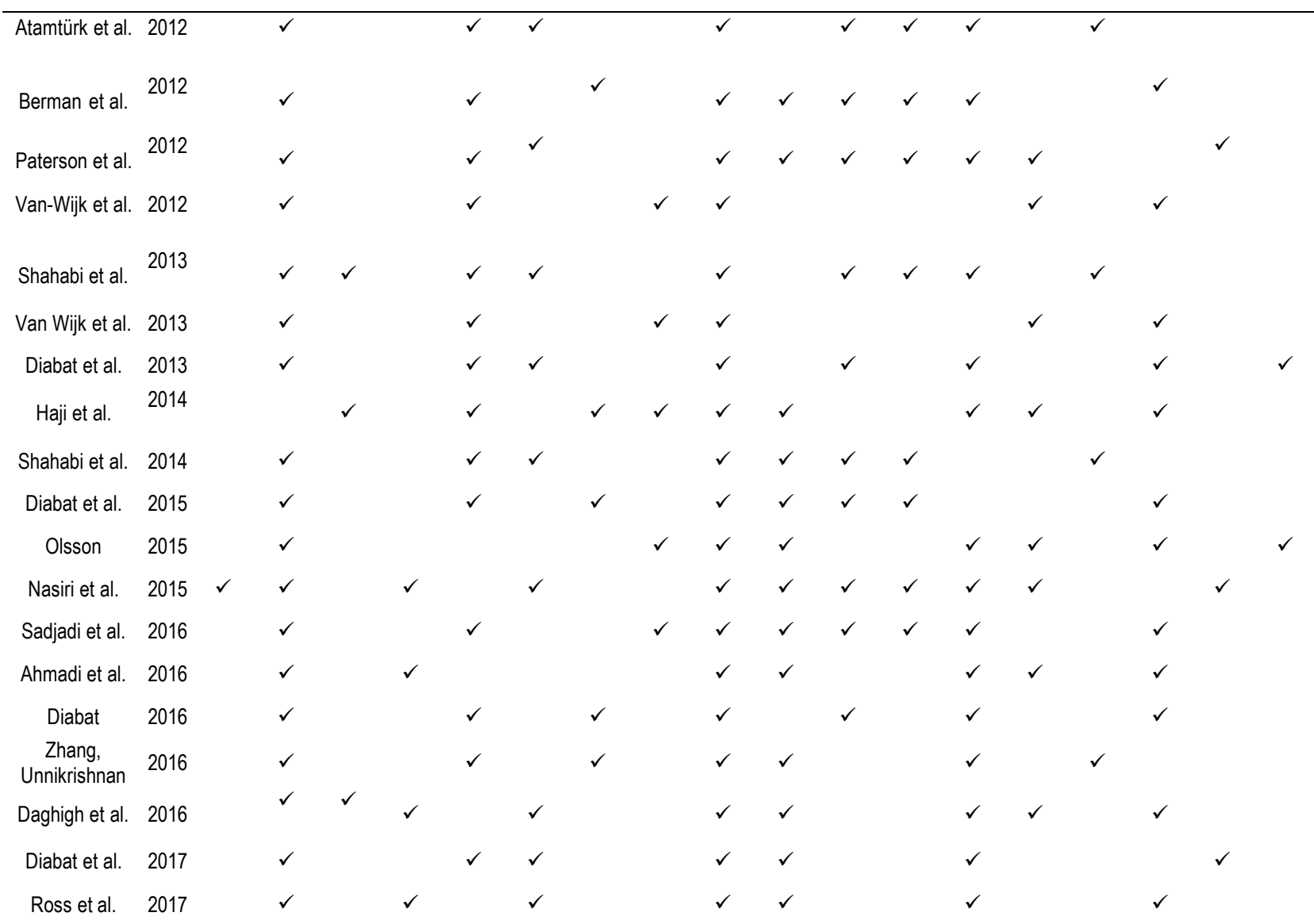




\begin{tabular}{|c|c|c|c|c|c|c|c|c|c|c|c|c|c|c|c|c|c|c|c|}
\hline \multirow[t]{2}{*}{ Authors } & \multirow[t]{2}{*}{ Year } & \multicolumn{3}{|c|}{ Location in } & \multicolumn{2}{|c|}{ Product } & \multicolumn{3}{|c|}{ Inventory Policy } & \multicolumn{5}{|c|}{ Inventory Cost } & \multicolumn{5}{|c|}{ Solution Approach } \\
\hline & & 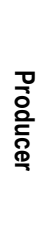 & 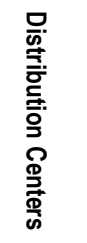 & 옿 & 즐 & $\frac{\mathscr{O}}{\bar{\Xi}}$ & $\begin{array}{l}\widehat{X ్ ర 0} \\
\underline{0}\end{array}$ & $\stackrel{\widehat{X}}{\exists}$ & $\begin{array}{l}\bar{\infty} \\
\stackrel{\vec{\omega}}{\vec{p}}\end{array}$ & 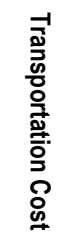 & 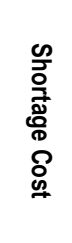 & 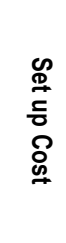 & 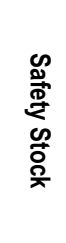 & 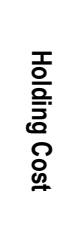 & 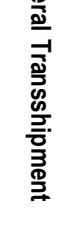 & 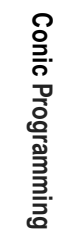 & 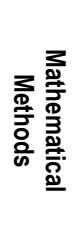 & 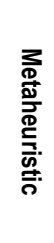 & 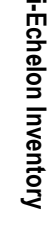 \\
\hline Feng et al. & 2017 & & $\checkmark$ & & & $\checkmark$ & & & $\checkmark$ & $\checkmark$ & $\checkmark$ & $\checkmark$ & $\checkmark$ & $\checkmark$ & $\checkmark$ & & $\checkmark$ & & \\
\hline Meissner, Senicheva & 2018 & & $\checkmark$ & & & $\checkmark$ & & & $\checkmark$ & $\checkmark$ & $\checkmark$ & & & $\checkmark$ & $\checkmark$ & & $\checkmark$ & & \\
\hline Van Wijk et al. & 2018 & & $\checkmark$ & & & $\checkmark$ & & & $\checkmark$ & $\checkmark$ & & & & & $\checkmark$ & & $\checkmark$ & & \\
\hline This paper & -.- & & $\checkmark$ & & & $\checkmark$ & $\checkmark$ & & & $\checkmark$ & $\checkmark$ & $\checkmark$ & $\checkmark$ & $\checkmark$ & $\checkmark$ & $\checkmark$ & & & $\checkmark$ \\
\hline
\end{tabular}

\section{MODEL DEVELOPMENT}

To obtain the model with lateral transshipment and multi-echelon inventory system, a supply chain system is assumed in which a producer intends to distribute the products among retailers via distribution centers. The aim of the model is to determine the number and location of distribution centers and their allocation to producer and retailers (i.e. direct connection) and to each other (i.e. lateral connection) under pre-defined inventory control policy. Inventory control system is characterized as a multi-echelon system and each active distribution center is assumed to be allocated to only one other active distribution centers or producers (i.e. unidirectional lateral transshipment). The notations and assumptions of the model are brought as follows.

\section{Sets:}

I Set of retailers indexed by $i$

J Set of distribution centers with indices $\mathrm{m}, \mathrm{j}$, and $k$. Meanwhile, $j=0$ represents the producer.

Parameter:

$h_{j} \quad$ Holding cost of each product per time unit at $\mathrm{j}^{\text {th }}$ distribution center

$A_{j} \quad$ Fixed ordering cost at $\mathrm{j}^{\text {th }}$ distribution center

$D_{j} \quad$ Mean of allocated demand at $\mathrm{j}^{\text {th }}$ distribution center

$V_{j} \quad$ Variance of allocated demand at $\mathrm{j}^{\text {th }}$ distribution center

$\mu_{i} \quad$ Mean of $\mathrm{i}^{\text {th }}$ retailer's demand

$\sigma_{i} \quad$ Standard deviation of $\mathrm{i}^{\text {th }}$ retailer's demand

$F_{j} \quad$ Fixed installation cost of $\mathrm{j}^{\text {th }}$ distribution center

$C_{j k} \quad$ Transportation cost of each product from $\mathrm{j}^{\text {th }}$ to $\mathrm{k}^{\text {th }}$ distribution centers

$P_{j i} \quad$ Transportation cost of each product from $\mathrm{j}^{\text {th }}$ distribution center to $\mathrm{i}^{\text {th }}$ retailer; $j \neq 0$

$L_{k j} \quad$ Lead time from $\mathrm{j}^{\text {th }}$ to $\mathrm{k}^{\text {th }}$ distribution centers

$z_{\alpha} \quad$ Standard normal deviate (i.e. $\mathrm{p}\left(\mathrm{z} \leq z_{\alpha}\right)=$ $\alpha)$

$\beta \quad$ Number of days per year

M A fixed positive integer number
Variables:

$\begin{array}{ll}y_{j i} & \begin{array}{l}\text { Binary variable: } 1 \text { if the } \mathrm{i}^{\text {th }} \text { retailer is } \\ \text { allocated to the } \mathrm{j}^{\text {th }} \text { distribution center, } 0 \\ \text { otherwise; }\end{array} \\ Z_{j k} \quad \begin{array}{l}\text { Binary variable: } 1 \text { if the } \mathrm{k}^{\text {th }} \text { distribution } \\ \text { center is allocated to the } \mathrm{j}^{\text {th }} \text { distribution } \\ \text { center, } 0 \text { otherwise; } \mathrm{k}=0 \text { demonstrates }\end{array} \\ \begin{array}{l}\text { producer. } \\ X_{j}\end{array} \quad \begin{array}{l}\text { Binary variable: } 1 \text { if the } \mathrm{j}^{\text {th }} \text { distribution } \\ \text { center is active, } 0 \text { otherwise. }\end{array}\end{array}$

Model Assumptions:

- Daily retailers' demands are independent across periods.

- Demand of $j^{\text {th }}$ distribution center is considered to follow the normal distribution with mean $\left(D_{j}\right)$ and variance $\left(V_{j}\right)$.

- Demand of $i^{\text {th }}$ retailer is considered to follow the normal distribution with mean $\left(\mu_{i}\right)$ and standard deviation $\left(\sigma_{i}\right)$.

- Retailers' order is considered independent from lateral distributors' order.

- Each retailer can be allocated to only one distribution center.

- Each distribution center can receive the products from only one other distribution center or producer and can deliver the products to at most one distribution center.

- If a distribution center is allocated to second distribution center, this second distribution center cannot be allocated to the third distribution center and should be definitely allocated to producer.

- $(R, Q)$ inventory control policy is used at distribution centers.

- Location decisions are made only for distribution centers and locations of producer and the location of retailers are already known.

- The lateral transshipment is unidirectional.

- The model is assumed with infinite capacity at distribution centers.

- The supply chain is designed for single item product.

- No shortages are allowed. 


\subsection{Modeling Description}

The model is developed based on minimizing total expected cost that consisting fixed cost of locating distribution centers, inventory control system costs and transportation costs. The cost functions are determined based on allocated demands to distribution centers and hence, this demand should be taken into account at each distribution center. In this section, in order to introduce the concept behind echelon-based systems, first the relations are developed for a simple two-echelon based model and then the relations are expanded into general model.

\subsubsection{Simple Two-Echelon Based Model}

Generally, each distribution center may encounter with two types of demand. The first type of demand is directly allocated by retailer and the second type is the lateral replenishment from other distribution centers.

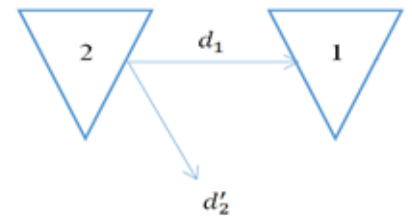

Figure 1 Two types of demands at each distribution center

Figure 1 illustrates these two types of demand in which the second distribution center, should replenish demand of the first distribution center (e.g. $\left.d_{l}\right)$ in lateral transshipment along with the demand which is directly receives from the retailer (e.g. $\left.d_{2}^{\prime}\right)$.

Since demands are assumed independent, the multiechelon inventory system should be redefined for lateral relations of distribution centers. As mentioned previously, based on this system, the echelon stock at each distribution center includes on-hand stock plus all downstream stocks of this center. Instead, echelon holding costs $\left(\mathrm{e}_{\mathrm{j}}\right)$ are used instead of traditional holding cost $\left(\mathrm{h}_{\mathrm{j}}\right)$ which means the value-added transmission of an item from an upstream to its downstream. In a two-echelon system, the echelon holding cost is defined as follows:

$$
\begin{aligned}
& e_{1}=h_{1}-h_{2} \\
& e_{2}=h_{2}
\end{aligned}
$$

In addition, based on optimal policy under multiechelon inventory system, batch quantities of upstream should be integer multiplier of their downstream quantities. In fact, in a two-echelon inventory system, we will have:

$$
Q_{2}=M Q_{1}
$$

where $M$ is a fixed positive integer number which is obtained as follows (Axsäter, 2015):

$$
\widehat{M}=\sqrt{\frac{A_{2} e_{1}}{A_{1} e_{2}}}
$$

As shown by Axsater (2015), $\widehat{M}$ has direct relation with ordering cost (A) and reverse relation with echelon holding cost (e). Since M should be an integer, the optimal $\mathrm{M}$ is incurred from equation (5):

$$
\left\{\begin{array}{l}
M=\lfloor\widehat{M}\rfloor \quad \text { if } \quad \frac{\widehat{M}}{\lfloor\widehat{M}\rfloor}<\frac{\lceil\widehat{M}\rceil}{\widehat{M}} \\
M=\lceil\widehat{M}\rceil \text { if } \frac{\widehat{M}}{\lfloor\widehat{M}\rfloor}>\frac{\lceil\widehat{M}\rceil}{\widehat{M}}
\end{array}\right.
$$

Where $L\rfloor$ and \lceil\rceil are respectively "floor" and "ceiling" rounding functions.

In special cases where $\widehat{M}<1$, the optimal value would be $\mathrm{M}=1$.

Then, in an inventory system with two-echelon, the inventory costs $\left(C_{j}^{e}\right)$ at each echelon including echelon holding and ordering costs (equations (6) and (7)) and at the entire system $\left(\mathrm{C}^{\mathrm{e}}\right)$ (equation (8)) are determined as follows:

$$
\begin{aligned}
& C_{1}^{e}=e_{1} \frac{Q_{1}}{2}+\frac{A_{1} \beta d_{1}}{Q_{1}} \\
& C_{2}^{e}=e_{2} \frac{M Q_{1}}{2}+\frac{A_{2} \beta d_{1}}{M Q_{1}} \\
& C^{e}=C_{1}^{e}+C_{2}^{e}=\left(e_{1}+M e_{2}\right) \frac{Q_{1}}{2} \\
&+\left(A_{1}+\frac{A_{2}}{M}\right) \frac{\beta d_{1}}{Q_{1}}
\end{aligned}
$$

Now, by assuming that the second distribution center encounters with the direct demand $\left(d_{2}^{\prime}\right)$ from retailer, the inventory cost associated with this direct demand $\left(C_{2}\right)$ would be as shown in equation (9):

$$
C_{2}=\frac{h_{2} Q_{2}}{2}+\frac{A_{2} \beta d_{2}^{\prime}}{Q_{2}}
$$

Where $Q_{2}$ represents the batch quantity of retailer's order.

Finally, the total cost $\left(C^{T}\right)$ from equations (8) and (9) will be equal to:

$$
\begin{aligned}
C^{T}=C^{e}+C_{2}= & \left(e_{1}+M e_{2}\right) \frac{Q_{1}}{2} \\
& +\left(A_{1}+\frac{A_{2}}{M}\right) \frac{\beta d_{1}}{Q_{1}} \\
& +\frac{h_{2} Q_{2}}{2}+\frac{A_{2} \beta d_{2}^{\prime}}{Q_{2}}
\end{aligned}
$$

Based on equation (10), the optimal value of batch quantities similar to any other EOQ model can be obtained as follows:

$$
\begin{aligned}
\frac{\partial C_{2}}{\partial Q_{2}}=0 \Rightarrow Q_{2}^{*}= & \sqrt{\frac{A_{2} \beta d_{2}^{\prime}}{2 h_{2}}} \Rightarrow C_{2} \\
& =\sqrt{2 A_{2} \beta d_{2}^{\prime} h_{2}}
\end{aligned}
$$




$$
\begin{aligned}
& \frac{\partial \mathrm{C}^{\mathrm{e}}}{\partial \mathrm{Q}_{1}}=0 \Rightarrow \mathrm{Q}_{1}^{*}=\sqrt{\frac{2\left(\mathrm{~A}_{1}+\frac{\mathrm{A}_{2}}{\mathrm{M}}\right) \beta d_{1}}{\mathrm{e}_{1}+\mathrm{Me}_{2}}} \\
& \Rightarrow C^{e}=\sqrt{2\left(A_{1}+\frac{A_{2}}{M}\right) \beta d_{1}\left(e_{1}+M e_{2}\right)}
\end{aligned}
$$

Finally, the optimal costs of inventory control system from equations (11) and (12) would be equal to:

$$
\begin{aligned}
& C^{T}=C^{e}+C_{2} \\
& =\sqrt{2 A_{2} \beta d_{2}^{\prime} h_{2}} \\
& +\sqrt{2\left(A_{1}+\frac{A_{2}}{M}\right) \beta d_{1}\left(e_{1}+M e_{2}\right)}
\end{aligned}
$$

\subsubsection{The General Model}

Now, let's generalize the concept to all distribution centers. For each pair of distribution center $(\mathrm{j}, \mathrm{k})$, if distribution center $\mathrm{j}$ can cover distribution center $\mathrm{k}$ with lateral transshipment, we will have:

$$
e_{j}=h_{j}
$$

$$
\begin{aligned}
& j, k \in J, \\
& j, k \neq 0 \\
& j, k \in J \text {, } \\
& j, k \neq 0 \\
& j, k \in J, \\
& j, k \in J \text {, } \\
& j, k \neq 0 \\
& S S_{j}=z_{\alpha} h_{j} \sqrt{V_{j} L_{0 j}}=z_{\alpha} h_{j} \sqrt{\sum_{i \in I} L_{0 j} \sigma_{i}^{2} y_{j i}} \quad \begin{array}{r}
\forall \\
j \in J, j \neq \\
0
\end{array}
\end{aligned}
$$$$
M_{j k}=\sqrt{\frac{A_{j} e_{k}}{A_{k} e_{j}}}
$$$$
C_{j}^{T}=\sqrt{2 A_{j} h_{j} \sum_{i \in I} \beta \mu_{i} y_{j i} Z_{0 j}}+\sqrt{\sum_{\substack{k \in J \\ k \neq j}} \sum_{i \in I} 2\left(A_{k}+\frac{A_{j}}{M_{j k}}\right)\left(e_{k}+M_{j k} e_{j}\right) \beta \mu_{i} y_{k i} Z_{j k}}
$$

Equations (14)-(16) are the same equations (1), (2), (4), which are redefined for each pair (j, k) of distribution centers. The relations can be rewritten for equations (5)(12) in similar way, but equation (13) is somehow different. Equation (17) represents this difference. In this equation, the first term illustrates the direct cost of replenishing the retailers. This term represents the demand mean of $\mathrm{j}^{\text {th }}$ distribution center based on sum of demand mean of all allocated retailers to this distribution center $\left(\mathrm{y}_{\mathrm{ji}}\right)$ under condition that this distribution center is activate $\left(\mathrm{z}_{0 \mathrm{j}}\right)$. The second term represents the lateral cost of supporting other distribution centers $(\mathrm{k})$ and allocated retailers to them $\left(\mathrm{y}_{\mathrm{ki}}\right)$.

Apart from above cost functions, the safety stock costs $\left(\mathrm{SS}_{\mathrm{j}}\right)$ should be taken into account. If the total variance of lead time demand in $\mathrm{j}^{\text {th }}$ distribution center is considered by $\mathrm{V}_{\mathrm{j}} \mathrm{L}_{0 \mathrm{j}}$, then the safety stock costs based on type-1 service level can be calculated as follows:

The "type 1" service level ensures the stock-outs would not happen within the lead time with maximum probability of $\alpha$. Equation (18) illustrates the amount of safety stock based on normal distribution of demands with this probability which is multiplied in holding cost of $\mathrm{j}^{\text {th }}$ distribution center. Meanwhile, the demand variance of this distribution center $\left(\mathrm{V}_{\mathrm{j}}\right)$ has been calculated based on sum of demand variance of allocated retailers $\left(\sigma_{i}^{2}\right)$ to this distribution center

\subsection{The Final Model}

Based on above modeling descriptions, the final model will be:

$$
\begin{aligned}
& \operatorname{Min} T C=\sum_{\substack{j \in J \\
j \neq 0}}\left[F_{j} X_{j}+\sqrt{2 A_{j} h_{j} \sum_{i \in I} \beta \mu_{i} y_{j i} Z_{0 j}}+\sqrt{\sum_{\substack{k \in J \\
k \neq j}}\left(2\left(A_{k}+\frac{A_{j}}{M_{j k}}\right)\left(e_{k}+M_{j k} e_{j}\right) \sum_{i \in I} \beta \mu_{i} y_{k i} Z_{j k}\right)}\right. \\
& +\sum_{i \in I} C_{0 j} \beta \mu_{i} y_{j i} Z_{0 j}+\sum_{\substack{k \in J \\
k \neq j}} \sum_{i \in I} C_{0 j} \beta \mu_{i} y_{k i} Z_{0 j} Z_{j k}+\sum_{\substack{k \in J \\
k \neq j}} \sum_{i \in I} C_{j k} \beta \mu_{i} y_{k i} Z_{j k}+\sum_{i \in I} P_{j i} \beta \mu_{i} y_{j i} \\
& \left.+z_{\alpha} h_{j} \sqrt{\sum_{i \in I} L_{0 j} \sigma_{i}^{2} y_{j i}}\right]^{k \neq j}
\end{aligned}
$$




$\begin{array}{ll}\sum_{\substack{j \in J \\ j \neq 0}} y_{j i}=1 & \forall j \in J, j \neq 0, i \\ y_{k i} \leq \sum_{\substack{j \in J \\ j \neq k}} Z_{j k} & \in I \\ X_{j}+X_{k} \geq 2 Z_{j k} & \forall j, k \in J, j \\ & \neq 0, j \neq k, i \\ Z_{j k}+Z_{k j} \leq 1 & \in I \\ \sum_{k \in J} Z_{j k} \leq Z_{0 j} & \neq j, k \in J, j \neq k \\ Z_{j k}=\{0,1\} & \forall j, k \in J, j \neq k \\ & \neq 0 \\ y_{j i}=\{0,1\} & \forall j, k \in J, j \neq k \\ X_{j}=\{0,1\} & \neq 0 \\ & \forall j, k \in J, j \neq k \\ & \neq 0 \\ & \forall j \in J, j \neq 0, i \\ & \in I \\ & \forall j \in J, j \neq 0\end{array}$

In objective function (19), the first term represents fixed installation cost for distribution centers, the second and third terms correspond to inventory costs in distribution centers, the next four terms represent the transportation costs from producer to distribution center, between distribution centers (directly and indirectly) and from distribution centers to retailers respectively. The last term represents safety stock costs. Constraints (20) guarantee that each retailer is allocated to exactly one distribution center. Constraints (21) ensure that each distribution center can only allocated to one another distribution center or producer. Constraints (22) state that, if a distribution center is allocated to another one, both centers must be definitely active. Constraints (23) prevent loop formation between two distribution centers. Constraints (24) explain that, each distribution center can receive the products from at most one other distribution center or producer and can deliver the products to at most one distribution center. Also, this set of constraints can simultaneously facilitate the chain management. Finally, constraints (25)-(27) enforce the integrality restrictions on the binary variables.

\section{SOLUTION APPROACH}

In this research, conic programming approach is used to solve the developed model (19)-(27). As mentioned in (Shahabi et al, 2014), the advantages of this method over other methods are in obtaining general optimal solution, shorter running time, getting away from special coding and ability to solve directly using standard optimization software packages such as CPLEX (Ben-Tal and Nemirovski, 2001).

In order to obtain the quadratic conic model, the objective function should be linear and constraints should be linear or quadratic (Atamtürk et al, 2012). But the developed model contains nonlinear terms in objective function. To linearize these terms (i.e. $y_{j i} Z_{0 j}, y_{k i} Z_{j k}, y_{k i} Z_{0 j}$ $\mathrm{Z}_{\mathrm{jk}}$ ) two new variables are defined as follow:

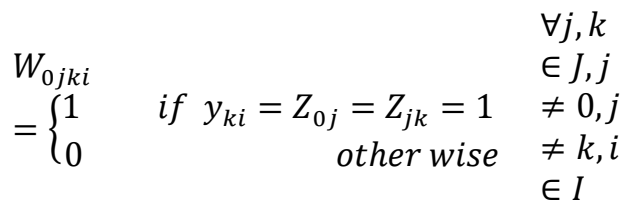

$$
\begin{aligned}
& W_{j k i}^{\prime} \quad \forall j, k \\
& =\left\{\begin{array}{rrr}
1 & \text { if } y_{k i}=Z_{j k}=1 & \in J, j \\
0 & \text { otherwise } & \neq k, i
\end{array}\right. \\
& \in I
\end{aligned}
$$

Meanwhile, the following constraints should be kept.

$$
\begin{array}{ll} 
& \forall j, k \epsilon J, k \\
W_{j k i}^{\prime} \leq y_{k i} & \neq 0, j \\
& \neq k, i \epsilon I \\
& \forall j, k \epsilon J, k \\
W_{j k i}^{\prime} \leq Z_{j k} & \neq 0, j \\
& \neq k, i \epsilon I \\
& \forall j, k \epsilon J, k \\
1+W_{j k i}^{\prime} \geq y_{k i}+Z_{j k} & \neq 0, j \\
& \neq k, i \epsilon I \\
W_{0 j k i} \leq y_{k i} & \forall j, k \epsilon J, j \\
& \neq k, j \neq k \\
& \neq 0, i \epsilon I \\
W_{0 j k i} \leq Z_{j k} & \forall j, k \epsilon J, j \\
& \neq k, j \neq k \\
W_{0 j k i} \leq Z_{0 j} & \neq 0, i \epsilon I \\
& \forall j, k \epsilon J, j \\
& \neq k, j \neq k \\
2+W_{0 j k i} \geq Z_{0 j}+Z_{j k} & \neq 0, i \epsilon I \\
& \forall j, k \epsilon J, j \\
& \neq k, j \neq k \\
& \neq 0, i \epsilon I \\
y_{k i} & \forall j, k \epsilon J, j \\
W_{0 j k i}, W_{j k i}^{\prime}=\{0,1\} & \neq k, j \neq k \\
& \neq 0, i \epsilon I
\end{array}
$$

By inserting the above constraints into the model, the objective function of the model will be changed as follows:

$$
\begin{aligned}
\operatorname{Min} T C=\sum_{\substack{j \in J \\
j \neq 0}}\left[F_{j} X_{j}+\sqrt{2 A_{j} h_{j} \sum_{i \in I} \beta \mu_{i} W_{0 j i}^{\prime}}+\sqrt{\sum_{\substack{k \in J \\
k \neq j}}\left(2\left(A_{k}+\frac{A_{j}}{M_{j k}}\right)\left(e_{k}+M_{j k} e_{j}\right) \sum_{i \in I} \beta \mu_{i} W_{j k i}^{\prime}\right)}+\sum_{i \in I} C_{0 j} \beta \mu_{i} W_{0 j i}^{\prime}\right. \\
\left.+\sum_{\substack{k \in J \\
k \neq j}} \sum_{0 j} \beta \mu_{i} W_{0 j k i}+\sum_{\substack{k \in J \\
k \neq j}} \sum_{\substack{i \in I\\
}} C_{j k} \beta \mu_{i} W_{j k i}^{\prime}+\sum_{i \in I} P_{j i} \mu_{i} \beta y_{j i}+z_{\alpha} h_{j} \sqrt{\sum_{i \in I} L_{0 j} \sigma_{i}^{2} y_{j i}}\right]
\end{aligned}
$$


Meanwhile, the objective function contains three nonlinear terms which should be linearized. So, three new positive variables are defined as shown in (39)-(41):

$$
\begin{array}{rlr}
t_{j}=\sqrt{2 A_{j} h_{j} \sum_{i \in I} \beta \mu_{i} W_{0 j i}^{\prime}} & \forall j, j \neq 0 \\
t_{j}^{\prime}=\sqrt{\left.\sum_{\left.\substack{k \in J \\
k \neq j} \sum_{i \in I} \beta \mu_{i} W_{j k i}^{\prime}\left(e_{k}+\frac{A_{j}}{M_{j k}}\right)\right)} M_{j k} e_{j}\right)} & \forall j, j \neq 0 \\
t_{j}^{\prime \prime}=\sqrt{\sum_{i \in I} L_{0 j} \sigma_{i}^{2} y_{j i}} & \forall j, j \neq 0
\end{array}
$$

Based on conic programming approach, by considering the nature of binary variables and substituting binary variables (i.e. $W_{0 j i}^{\prime}, W_{j k i}^{\prime}, y_{j i}$ ) with their quadratic forms, the following equations would be obtained:

$$
\begin{aligned}
& 2 A_{j} h_{j} \sum_{i \in I} \beta \mu_{i}\left(W_{0 j i}^{\prime}\right)^{2} \leq t_{j}^{2} \quad \begin{array}{ll}
\forall j, j \\
\end{array} \\
& \sum_{\substack{k \in J \\
k \neq j}}\left(\begin{array}{c}
2\left(A_{k}+\frac{A_{j}}{M_{j k}}\right) \\
\left(e_{k}+M_{j k} e_{j}\right) \sum_{i \in I} \beta \mu_{i}\left(W_{0 j i}^{\prime}\right)^{2}
\end{array}\right) \quad \begin{array}{l}
\forall j, j \\
\neq 0
\end{array} \\
& \leq t_{j}^{\prime 2}
\end{aligned}
$$

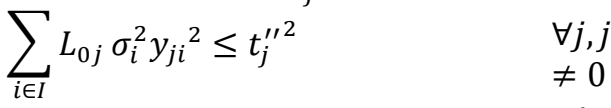

$$
\begin{aligned}
& t_{j}, t_{j}^{\prime}, t_{j}^{\prime \prime} \geq 0 \quad \in J, j
\end{aligned}
$$

By inserting new variables (39)-(41) and equations (42)-(45), the model can be reformulated into the following conic programming form:

$$
\begin{aligned}
& \operatorname{Min} T C=\sum_{\substack{j \in J \\
j \neq 0}}\left[F_{j} X_{j}+t_{j}+t_{j}^{\prime}+\sum_{i \in I} C_{0 j} \beta \mu_{i} W_{0 j i}^{\prime}\right. \\
& +\sum_{k \in J} \sum_{i \in I} C_{0 j} \beta \mu_{i} W_{0 j k i} \\
& +\sum_{\substack{k \in J \\
k \neq j}}^{k \neq j} \sum_{i \in I} C_{j k} \beta \mu_{i} W_{j k i}^{\prime}+\sum_{i \in I} P_{j i} \mu_{i} y_{j i} \\
& \left.+z_{\alpha} h_{j} t_{j}^{\prime \prime}\right] \\
& \sum_{\substack{j \in J \\
j \neq 0}} y_{j i}=1 \\
& \forall j \in J, j \neq 0, i \\
& \in I
\end{aligned}
$$

$$
\begin{aligned}
& y_{k i} \leq \sum_{\substack{j \in J \\
j \neq k}} Z_{j k} \\
& X_{j}+X_{k} \geq 2 Z_{j k} \\
& Z_{j k}+Z_{k j} \leq 1 \\
& \sum_{k \in J} Z_{j k} \leq Z_{0 j} \\
& 2 A_{j} h_{j} \sum_{i \in I} \beta \mu_{i}\left(W_{0 j i}^{\prime}\right)^{2} \leq t_{j}^{2} \\
& \sum_{\substack{k \in J \\
k \neq j}}\left(\begin{array}{c}
2\left(A_{k}+\frac{A_{j}}{M_{j k}}\right)\left(e_{k}+M_{j k} e_{j}\right) \\
\sum_{i \in I} \beta \mu_{i}\left(W_{0 j i}^{\prime}\right)^{2}
\end{array}\right) \forall j \in J, j \neq 0 \\
& \leq t_{j}^{\prime 2} \\
& \sum_{i \in I} L_{0 j} \sigma_{i}^{2} y_{j i}^{2} \leq t_{j}^{\prime 2} \\
& W_{j k i}^{\prime} \leq y_{k i} \\
& W_{j k i}^{\prime} \leq Z_{j k} \\
& 1+W_{j k i}^{\prime} \geq y_{k i}+Z_{j k} \\
& W_{0 j k i} \leq y_{k i} \\
& W_{0 j k i} \leq Z_{j k} \\
& W_{0 j k i} \leq Z_{0 j} \\
& 2+W_{0 j k i} \geq Z_{0 j}+Z_{j k}+y_{k i} \\
& W_{0 j k i}, W_{j k i}^{\prime}=\{0,1\} \\
& Z_{j k}=\{0,1\} \\
& y_{j i}=\{0,1\} \\
& X_{j}=\{0,1\} \\
& t_{j}, t_{j}^{\prime}, t_{j}^{\prime \prime} \geq 0 \\
& \forall j, k \in J, j \neq 0, j \\
& \neq k, i \in I \\
& \forall j, k \in J, j \neq k \\
& \neq 0 \\
& \forall j, k \in J, j \neq k \\
& \neq 0 \\
& \forall j, k \in J, j \neq k \\
& \neq 0 \\
& \forall j \in J, j \neq 0 \\
& \forall j \in J, j \neq 0 \\
& \forall j, k \in J, k \neq 0, j \\
& \neq k, i \in I \\
& \forall j, k \in J, k \neq 0, j \\
& \neq k, i \in I \\
& \forall j, k \in J, k \neq 0, j \\
& \neq k, i \in I \\
& \forall j, k \in J, j \neq k, j \\
& \neq k \neq 0, i \in I \\
& \forall j, k \in J, j \neq k, j \\
& \neq k \neq 0, i \in I \\
& \forall j, k \in J, j \neq k, j \\
& \neq k \neq 0, i \in I \\
& \forall j, k \in J, j \neq k, j \\
& \neq k \neq 0, i \in I \\
& \forall j, k \in J, j \neq k, j \\
& \neq k \neq 0, i \in I \\
& \forall j, k \in J, j \neq k \\
& \neq 0 \\
& \forall j \in J, j \neq 0, i \\
& \in I \\
& \forall j \in J, j \neq 0 \\
& \forall j \in J, j \neq 0
\end{aligned}
$$

\section{NUMERICAL RESULTS}

In this section, computational results will be presented for three different data sets with 5, 8 and 10 potential distribution centers and with sizes ranging from 5 to 100 retailers. For simplicity, each instance has been represented by $(d ; r)$ where the former $(d)$ is number of potential distribution centers and the latter $(r)$ is number of retailers. The experiments were conducted on a computer system with an Intel Core i5 2.6 GHz processor and $8 \mathrm{~GB}$ RAM. The models were run in GAMS. Note that the model is a mixed integer conic quadratic programs which CPLEX guarantees global optimality for these models. Therefore, the CPLEX 12 solver was used to solve the instances. The parameters have been generated with uniform distribution as shown in Table 2. 
Table 2 Numerical values of parameters

\begin{tabular}{|c|c|}
\hline Parameter & Value \\
\hline$h_{\mathrm{j}}:$ Per unit holding cost & $\mathrm{U}(1,5)$ \\
\hline$A_{j}$ : Fixed ordering cost & $\mathrm{U}(200,650)$ \\
\hline $\mathrm{D}_{\mathrm{i}}$ : Mean of allocated demand & $\mathrm{U}(20,60)$ \\
\hline $\mathrm{V}_{\mathrm{j}}$ : Variance of allocated demand & $\mathrm{U}(1,5)$ \\
\hline $\mathrm{F}_{\mathrm{j}:}$ Fixed installation cost & $U(20000,80000)$ \\
\hline $\mathrm{C}_{\mathrm{jk}}$ : Lateral transportation cost & $\mathrm{U}(0.2,2)$ \\
\hline$P_{\mathrm{ji}}:$ Direct transportation cost & $\mathrm{U}(0.2,2)$ \\
\hline Lkj: Order lead time & $\mathrm{U}(10,40)$ \\
\hline$z_{\alpha}:$ Standard normal deviate & 3 \\
\hline$\beta:$ Number of days per year & 250 \\
\hline
\end{tabular}

It should be noted that $M_{j k}$ is a dependent parameter which is calculated by equation (16). In addition, defining a reference model is the way to demonstrate the superiority of the developed model in comparison with previous studies. So, the Shen's model (2003) - as the main basic model of this study - was selected as reference model. Each instance was solved for both developed and reference models and results were reported in Tables 3, 4 and 5. Meanwhile, the difference of results $(\delta)$ and running times were demonstrated. The difference is an important measure for illustrating performance improvement of the model and consequently model validation.

Table 3 Numerical results for instances with 5 distribution centers

\begin{tabular}{llllllll}
\hline & $\mathbf{( 5 ; 5 )}$ & $\mathbf{( 5 ; 1 0 )}$ & $\mathbf{( 5 ; 1 5 )}$ & $\mathbf{( 5 ; 2 0 )}$ & $\mathbf{( 5 ; 3 0 )}$ & $\mathbf{( 5 ; 5 0 )}$ & $\mathbf{( 5 ; 1 0 0 )}$ \\
\hline Developed Model (Obj. Fun.) & 76183 & 135464 & 175003 & 235360 & 299624 & 409462 & 724001 \\
Reference Model (Obj. Fun.) & 76183 & 135464 & 180410 & 245420 & 329145 & 468968 & 915407 \\
Difference ( $\boldsymbol{\delta})$ & 0 & 0 & 5407 & 10060 & 29521 & 59506 & 191406 \\
CPLEX time (Sec) & 0.14 & 0.22 & 0.25 & 1.72 & 3.01 & 8.27 & 48.27 \\
\hline
\end{tabular}

Table 4 Numerical results for instances with 8 distribution centers

\begin{tabular}{llllllll}
\hline & $\mathbf{( 8 ; 5 )}$ & $\mathbf{( 8 ; 1 0 )}$ & $\mathbf{( 8 ; 1 5 )}$ & $\mathbf{( 8 ; 2 0 )}$ & $\mathbf{( 8 ; 3 0 )}$ & $\mathbf{( 8 ; 5 0 )}$ & $\mathbf{( 8 ; 1 0 0 )}$ \\
\hline Developed Model (Obj. Fun.) & 95904 & 140619 & 170119 & 210381 & 276397 & 371481 & 655254 \\
Reference Model (Obj. Fun.) & 95904 & 140689 & 180174 & 245495 & 334655 & 463981 & 879249 \\
Difference ( $\mathbf{\delta})$ & 0 & 70 & 10055 & 35114 & 58258 & 92500 & 223995 \\
CPLEX time (Sec) & 0.42 & 1.15 & 4.82 & 9.15 & 20 & 73 & 1459 \\
\hline
\end{tabular}

Table 5 Numerical results for instances with 10 distribution centers

\begin{tabular}{llllllll}
\hline & $(\mathbf{1 0 ; 5})$ & $\mathbf{( 1 0 ; 1 0 )}$ & $\mathbf{( 1 0 ; 1 5 )}$ & $\mathbf{( 1 0 ; 2 0 )}$ & $\mathbf{( 1 0 ; 3 0 )}$ & $\mathbf{( 1 0 ; 5 0 )}$ & $\mathbf{( 1 0 ; 1 0 0 )}$ \\
\hline Developed Model (Obj. Fun.) & 95847 & 135675 & 156550 & 190174 & 255997 & 354723 & 627101 \\
Reference Model (Obj. Fun.) & 95847 & 154073 & 186203 & 239793 & 345344 & 493430 & 865146 \\
Difference (ס) & 0 & 18398 & 29653 & 49619 & 89347 & 138707 & 238045 \\
CPLEX time (Sec) & 0.5 & 5.48 & 13 & 34 & 119 & 668 & 2435 \\
\hline
\end{tabular}

As observed in Tables 3, 4 and 5, the results represent non-negative differences in all three tables. In small size instances, the same values were observed that is generally quite expected; since in these instances, the developed model behaves such as the reference model. In contrast, the difference is noticeable in medium and largesized instances which confirms the better performance of the developed location-inventory model considering multi echelon inventory system and lateral transshipment.

In addition, it is so important to check the effect of increase in number of potential distribution centers and retailers on solution improvement and running time.

Figures 2 - 5 represent these analyses.

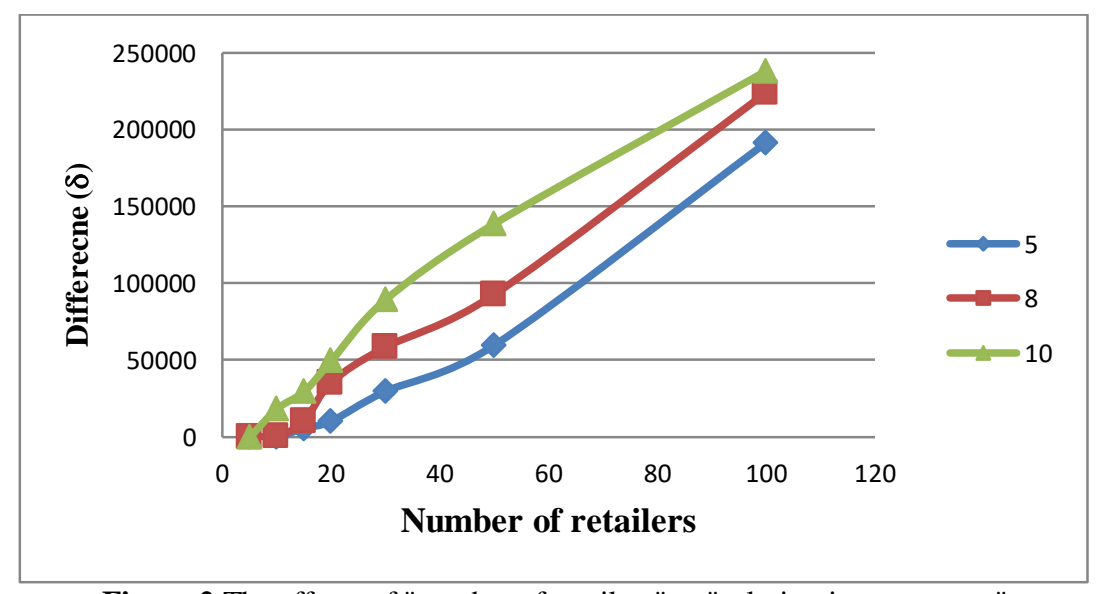

Figure 2 The effects of "number of retailers" to "solution improvement" 


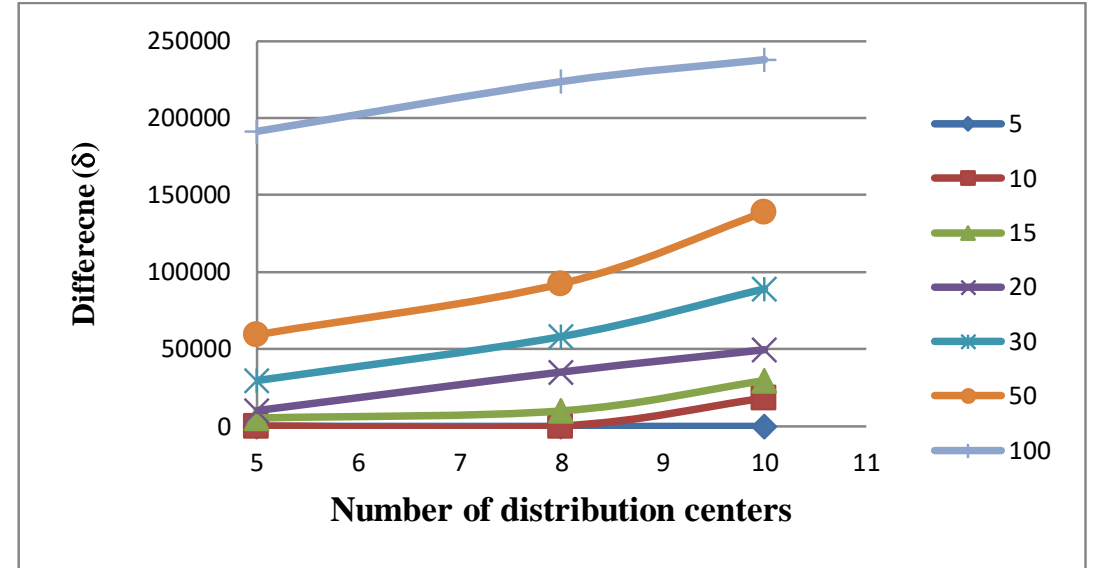

Figure 3 The effects of "number of distribution centers" to "solution improvement"

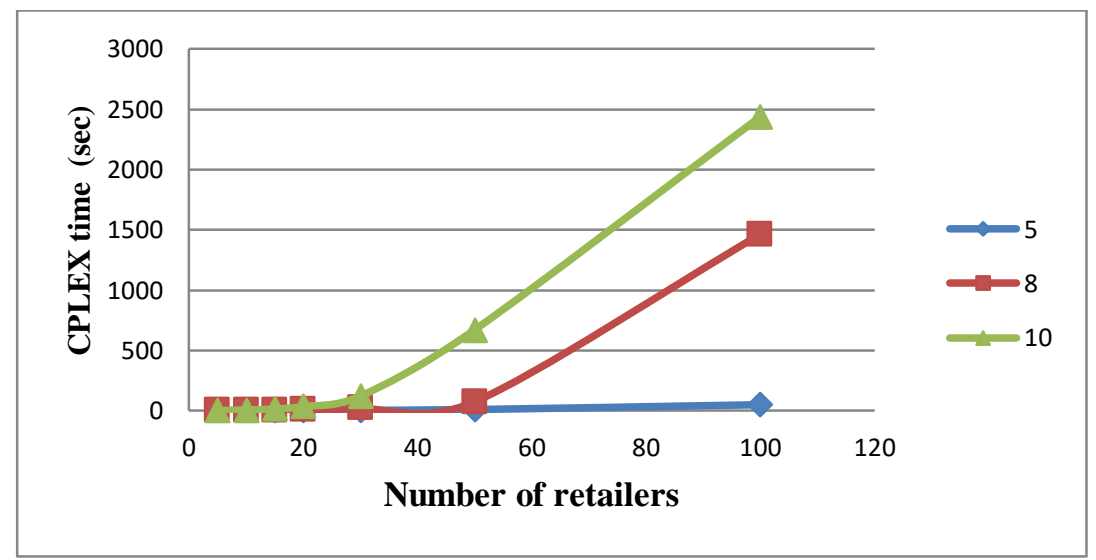

Figure 4 The effects of "number of retailers" to "running time"

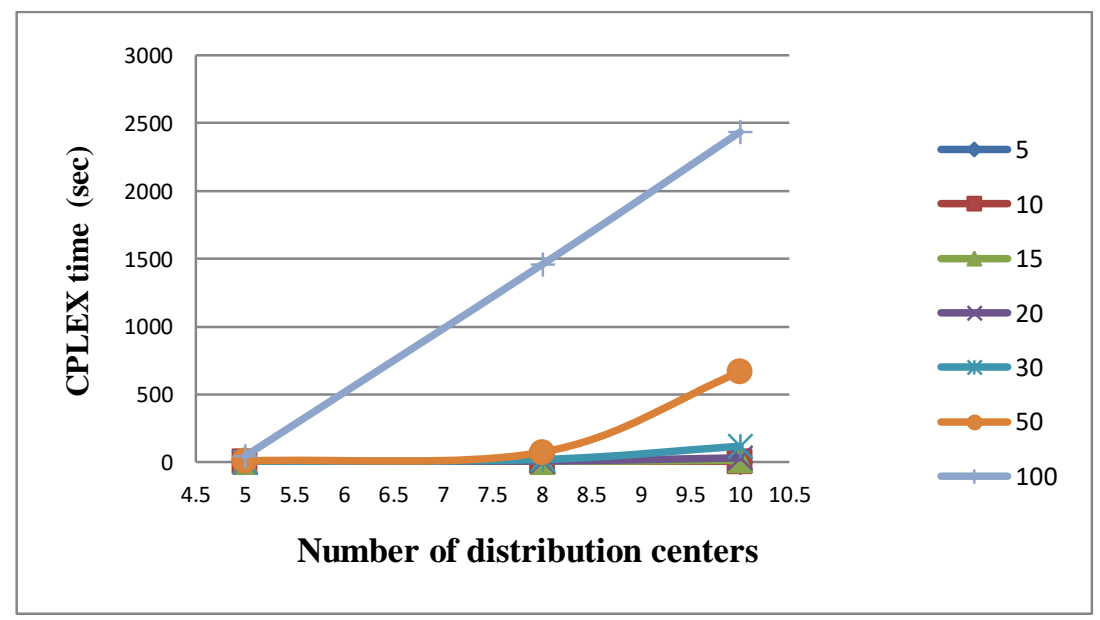

Figure 5 The effects of "number of distribution centers" to "running time"

Figure 2 compares the effect of increasing in number of retailers in solution improvement in three data sets (with 5,8 and 10 potential distribution centers). It should be noted that growth in retailer numbers leads to more demands which in turn increases the possibility of lateral allocations and at result improves the performance of developed model in comparison to the reference model. Meanwhile, as can be seen, all three diagrams have (more or less) the similar slopes, that demonstrates the normal behavior of the model in the face of increasing the number of retailers.

The opposite situation is shown in Figure 3. This time, the effect of increasing in number of potential distribution centers are compared in different sizes of retailers. However, the results are the same. More distribution centers will increase more lateral allocations and at result, more improvement is achieved. Again, diagrams with similar slopes at intervals corresponding to the size of retailers are observed that indicate the normal behavior of the model in the face of increasing the number of potential distribution centers.

In Figure 4, the effect of increasing in number of retailers in CPU running time is reported. As predicted, in small and medium-sized instances the running time is tiny due to the use of the conic programming approach in solving process. In large scale instances, the time is non-linearly increased. It should be noted that increasing in running time 
is essentially related to the increasing in number of nonlinear variables in quadratic form and also additional variables used in reformulation of final model.

Also, Figure 5 depicts the effect of increasing in number of potential distribution centers in CPU running time. Again, increasing in running time, especially in large scale instance, is the result of additional nodes and arcs in the distribution network of the problem induced by increasing the number of distribution centers.

\section{CONCLUSION}

In this paper, an integrated location-inventory model with unidirectional lateral transshipments and multiechelon inventory system has been presented. The aim is to determine the number and location of distribution centers and their allocation to producer and retailers (i.e. direct connection) and to each other (i.e. lateral connection) under multi-echelon inventory control policies. For this purpose, first the stock level of distribution centers is determined based on echelon inventory concept and then total inventory (i.e. holding and ordering) cost is calculated. Meanwhile, the "Type 1" service level measure is used to determine safety stock costs. Finally, by adding direct and lateral transportation costs and fixed installation costs, the objective function has been completed in the form of mixed integer non-linear programming model (MINLP). The model is then reformulated to conic programming to exploit the recent advances made in solving conic programs.

To evaluate the performance of developed model, three set of instances with different number of distribution centers and different number of retailers are considered. The outputs are compared with the reference model (i.e. the model without contributions) and the differences are reported at each instance. The results demonstrate the superiority of the developed model especially in medium and large-scale instances.

This research can be extended in several way such as using different inventory policies, using stochastic lead times, using other statistical distributions (e.g. Poisson distribution) for demand uncertainty and developing a model based on queueing approach, extending the model to inventory-location-routing with multiple products and different transportation modes, using other approaches to deal with uncertainty (e.g. fuzzy mathematical programming and robust optimization) and so on. Meanwhile, the model can be broadened by relaxing assumptions of this study; for example, instead of using one-to-one lateral allocation of distribution centers, one-tomany allocations can be used or instead of one producer, multiple producers can be defined and modeled and also four or more echelons of supply chain can be considered while locating-allocating decisions are made in all echelons.

\section{REFERENCES}

Abu Alhaj, M., Svetinovic, D., \& Diabat, A. (2016). A carbonsensitive two-echelon-inventory supply chain model with stochastic demand. Resources, Conservation and Recycling 108, pp. 82-87.

Ahmadi, G., Torabi, S. A., \& Tavakkoli-Moghaddam, R. (2016). A bi-objective location-inventory model with capacitated transportation and lateral transshipments. International Journal of Production Research 54(7), pp. 2035-2056.

Atamtürk, A., Berenguer, G., \& Shen, Z. J. (2012). A Conic Integer Programming Approach to Stochastic Joint Location-Inventory Problems. Operations Research 60, pp. 366-381.

Axsäter, S. (2015). Inventory Control. 3rd ed. Springer: New York.

Ben-Tal, A., \& Nemirovski, A. (2001). Lectures on modern convex optimization: Analysis, Algorithms and Engineering Applications. SIAM Publisher: Philadelphia.

Berman, O., Krass, D., \& Tajbakhsh, M. M. (2012). A coordinated location-inventory model. European Journal of Operational Research 217, pp. 500-508.

Daghigh, R., Jabalameli, M.S., Bozorgi Amiri, A., \& Pishvaee, M.S. (2016) A multi-objective location-inventory model for 3PL providers with sustainable considerations under uncertainty. International Journal of Industrial Engineering Computations 7, pp. 615-634.

Daskin, M. S., Coullard, C. R., \& Shen, Z. J. (2002). An inventory-location model: Formulation, solution algorithm and computational results. Annals of Operations Research 110, pp. 83-106.

Diabat, A., Richard, J. P., \& Codrington, C. (2013). A Lagrangian relaxation approach to simultaneous strategic and tactical planning in supply chain design. Annals of Operations Research 203(1), pp. 55-80.

Diabat, A., Battaïa, O., \& Nazzal, D. (2015). An improved Lagrangian relaxation-based heuristic for a joint locationinventory problem. Computers and Operations Research 61 , pp. 170-178.

Diabat, A., \& Theodorou, E. (2015). A location-inventory supply chain problem: Reformulation and piecewise linearization. Computers and Industrial Engineering 90, pp. 381-389.

Diabat, A. (2016). A capacitated facility location and inventory management problem with single sourcing. Optimization Letters 10(7), pp. 1577-1592.

Diabat, A., Dehghani, E., \& Jabbarzadeh, A. (2017). Incorporating location and inventory decisions into a supply chain design problem with uncertain demands and lead times. Journal of Manufacturing Systems 43(1), pp. 139-149.

Feng, P., Fung, R.Y.K. \& Wu, F. (2017). Preventive transshipment decisions in a multi-location inventory system with dynamic approach. Computers \& Industrial Engineering 104, pp. 1-8.

Haji, R., Tayebi, H., \& Jeddi, B. G. (2014). One-for-one-period ordering policy for inventory systems with unidirectional lateral transshipments. The International Journal of Advanced Manufacturing Technology 74, pp. 1159-1166.

Hillier, F. S., \& Lieberman, G. J. (2015). Introduction to Operations Research, $10^{\text {th }}$ Ed. Chapter 18.5. McGraw-Hill: New York.

Maass, K. L., Daskin, M. S., \& Shen, S. (2016). Mitigating Hard Capacity Constraints with Inventory in Facility Location Modeling. IIE Transactions 48(2), pp. 120-133.

Mak, H. Y., \& Shen, Z. J. (2009). A two-echelon inventorylocation problem with service considerations. Naval Research Logistics 56, pp. 730-744.

Meissner, J., \& Senicheva, O.V. (2018). Approximate dynamic programming for lateral transshipment problems in multilocation inventory systems. European Journal of Operational Research 265(1), pp. 49-64

Nasiri, G.R., Ghaffari, N., \& Davoudpour, H. (2015). Locationinventory and shipment decisions in an integrated distribution system: an efficient heuristic solution. European Journal of Industrial Engineering 9(5), pp. 613637. 
Olsson, F. (2015) Emergency lateral transshipments in a twolocation inventory system with positive transshipment lead times. European Journal of Operational Research 242(2), pp. 424-433.

Ozsen, L., Daskin, M. S., \& Coullard, C. (2008). Capacitated facility location model with risk pooling Networks. Naval Research Logistics 55(4), pp. 295-312.

Paterson, C., Teunter, R., \& Glazebrook, K. (2012). Enhanced lateral transshipments in a multi-location inventory system. European Journal of Operational Research 221, pp. 317327.

Puga, M. S., \& Tancrez, J. S. (2017). A heuristic algorithm for solving large location-inventory problems with demand uncertainty. European Journal of Operational Research 259(2), pp. 413-423.

Ross, A., Khajehnezhad, M., Otieno, W., \& Aydas, O. (2017). Integrated location-inventory modelling under forward and reverse product flows in the used merchandise retail sector: A multi-echelon formulation. European Journal of Operational Research 259(2), pp. 664-676.

Sadjadi, S. J., Makui, A., Dehghani, E., \& Pourmohammad, M. (2016). Applying queuing approach for a stochastic location-inventory problem with two different mean inventory considerations. Applied Mathematical Modelling 40(1), pp. 578-596.

Shahabi, M., Akbarinasaji, S., Unnikrishnan, A., \& James, R. (2013). Integrated Inventory Control and Facility Location Decisions in a Multi-Echelon Supply Chain Network with Hubs. Networks and Spatial Economics 13, pp. 497-514.
Shahabi, M., Unnikrishnan, A., Jafari-Shirazi, E., \& Boyles, S. D. (2014). A three level location-inventory problem with correlated demand. Transportation Research Part B: Methodological 69, pp. 1-18.

Shen, Z. J., Coullard, C., \& Daskin, M. S. (2003). A joint locationinventory model. Transportation Science 37, pp. 40-55.

Shen, Z. J. (2000). Efficient algorithms for various supply chain problems. Dissertation: Northwestern University.

Sherbrooke, G. (2004).Optimal Inventory Modeling of Systems: Multi-Echelon Techniques. $2^{\text {nd }}$ ed. Springer: New York.

Van-Wijk, A. C. C., Adan, I. J. B. F., \& Van-Houtum, G. J. (2012). Approximate evaluation of multi-location inventory models with lateral transshipments and hold back levels. European Journal of Operational Research 218, pp. 624635.

Van-Wijk, A. C. C., Adan, I. J. B. F., \& Van-Houtum, G. J. (2013). Optimal allocation policy for a multi-location inventory system with a quick response warehouse. Operations Research Letters 41, pp. 305-310.

Van-Wijk, A. C. C., Adan, I. J. B. F., \& Van-Houtum, G. J. (2018). Optimal lateral transshipment policies for a two location inventory problem with multiple demand classes. European Journal of Operational Research, 272(2), pp. 481-495.

Zhang, Z.H., \& Unnikrishnan, A. (2016). A coordinated locationinventory problem in closed-loop supply chain. Transportation Research Part B: Methodological 89, pp. 127-148.

Mohammad Reza Gholamian is an Associate Professor in School of Industrial Engineering at the Iran University of Science and Technology (IUST), Tehran, Iran. He received his M.S. degree in Industrial Engineering from Isfahan University of Technology (IUT), Isfahan in 1998 and obtained Ph.D. in Industrial Engineering from Amirkabir University of Technology (AUT), Tehran in 2005. Presently he is faculty member of Systems Engineering Group in School of Industrial Engineering and is actively engaged in conducting Academic, Research and Development Programs in the field of Industrial Engineering. He has contributed more than 172 research papers to many national and international journals and conferences. Besides this, he has published 5 books by reputed publishers. His research interests are in the areas of Inventory Models, Supply Chain Network Design and Multi-Criteria Decision Making.

Mustafa Nasri is an M.S. Graduate student in School of Industrial Engineering at the Iran University of Science and Technology (IUST), Tehran, Iran. His research interests include Facility Design, Supply Chain Management and Inventory Models. 